\title{
Erratum
}

\section{Production and decay of the Standard Model Higgs boson at LEP200}

\author{
Eilam Gross ${ }^{1}$, Bernd A. Kniehl ${ }^{2}$, Gustavo Wolf ${ }^{1}$ \\ ${ }^{1}$ Department of Particle Physics, Weizmann Institute of Science, Rehovot 76100, Israel \\ 2 II. Institut für Theoretische Physik, Universität Hamburg, Luruper Chaussee 149, D-22761 Hamburg, Germany
}

Received: 3 November 1994

\section{Z. Phys. C 63, 417-425 (1994)}

There is a typographical error in the expression for $\Gamma(H \rightarrow$ $g g$ ) listed in (23): The power of $\pi$ appearing in the denominator on the right-hand side should be 3 instead of 2 . The correct formula may be found, e.g., in (3.40) of [12]. In the computer program that was created for the purpose of this article, this very formula was implemented wrongly, too: There $\pi$ appeared with the power 5 instead of 3 . As a consequence, Figs. 9 and 10 and some entries in Tables 3-5 are erroneous. We thank G. Crosetti for noticing this. Further-

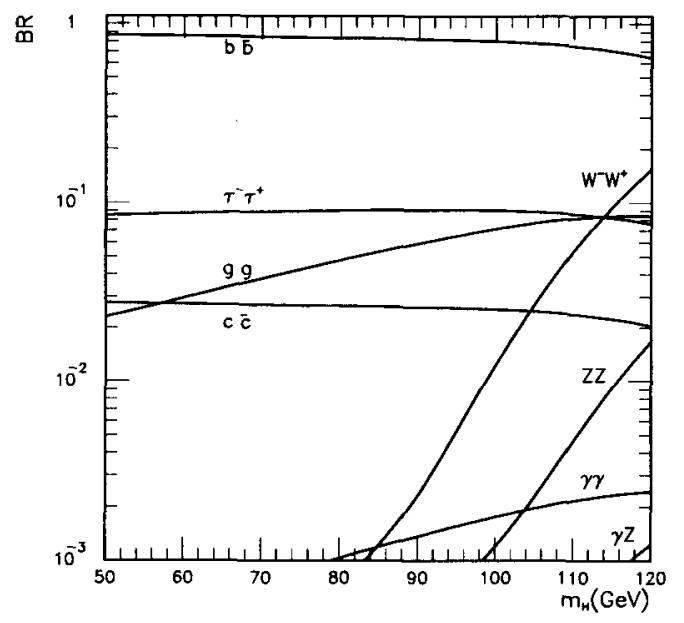

Fig. 9. Branching fractions of the Higgs boson in the $m_{H}$ window relevant for LEPI and LEP200. All radiative corrections discussed in the text are included more, the contributions due to the first five quark flavours are neglected in (23), which is an unnecessary simiplification and gives an unsatisfactory approximation. We thank P. Janot for pointing this out. (23) should be replaced by the exact one-loop formula, which is listed, e.g., in (3.39) of [12]. We choose to evaluate this formula using the quark pole masses. The revised figures and tables are presented below.
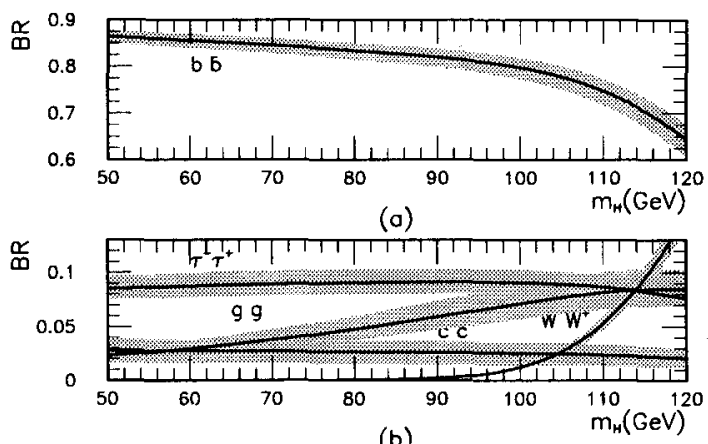

(b)

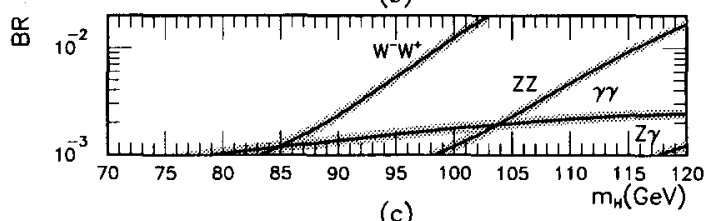

Fig. 10. Branching ratios of the Higgs-boson decays to (a) $b \bar{b}$, (b) $\tau^{+} \tau^{-}$, $g g, c \bar{c}, W^{*} W^{*}$, (c) $\gamma \gamma, \gamma Z$, and $Z^{*} Z^{*}$ versus $m_{H}$. The bands indicate the systematic errors 
Table 3. Branching fractions (in \%) of the Higgs boson in the $m_{H}$ window relevant for LEPI and LEP200. All radiative corrections discussed in the text are included

\begin{tabular}{llllllll}
\hline$m_{H}[\mathrm{GeV}]$ & $b \bar{b}$ & $\tau^{+} \tau^{-}$ & $c \bar{c}$ & $g g$ & $W^{*} W^{*}$ & $Z^{*} Z^{*}$ & $\gamma \gamma$ \\
\hline \hline 50 & 86.4 & 8.5 & 2.8 & 2.3 & - & - & - \\
\hline 60 & 85.6 & 8.7 & 2.8 & 2.9 & - & - & - \\
\hline 70 & 84.6 & 8.9 & 2.7 & 3.8 & - & - & - \\
\hline 80 & 83.3 & 9.0 & 2.7 & 4.8 & 0.1 & - & 0.1 \\
\hline 90 & 82.0 & 9.1 & 2.6 & 5.9 & 0.2 & - & 0.2 \\
\hline 100 & 79.8 & 9.1 & 2.5 & 7.1 & 1.2 & 0.1 & 0.2 \\
\hline 110 & 74.7 & 8.7 & 2.5 & 8.2 & 5.3 & 0.5 & 0.2 \\
\hline \hline
\end{tabular}

Table 5. Effects of the uncertainties in $M_{q}$ and $\alpha_{S}\left(m_{Z}\right)$ on the various Higgs branching ratios for $m_{H}=80.0 \mathrm{GeV}$

\begin{tabular}{|c|c|c|c|c|c|}
\hline Decay Mode & $B R[\%]$ & $\begin{array}{l}M_{b}= \\
(4.7 \pm 0.2) \\
\mathrm{GeV}\end{array}$ & $\begin{array}{l}M_{c}= \\
(1.45 \pm 0.05) \\
\mathrm{GeV}\end{array}$ & $\begin{array}{l}\alpha_{S}\left(m_{Z}\right)= \\
0.123 \pm 0.006\end{array}$ & $\begin{array}{l}M_{t}= \\
(165 \pm 35) \\
\mathrm{GeV}\end{array}$ \\
\hline$H \rightarrow \bar{b} \bar{b}$ & $\overline{83.3}$ & $\begin{array}{l}+1.4 \\
-1.6\end{array}$ & $\begin{array}{l}+0.3 \\
-0.3\end{array}$ & $\begin{array}{l}+0.7 \\
-1.0\end{array}$ & $\begin{array}{l}+0.1 \\
-0.1\end{array}$ \\
\hline$H \rightarrow c \bar{c}$ & 2.7 & $\begin{array}{l}+0.3 \\
{ }_{-0.2}\end{array}$ & $\begin{array}{l}+0.4 \\
-0.4\end{array}$ & $\begin{array}{l}+0.9 \\
-1.0\end{array}$ & $\begin{array}{l}+0.0 \\
+0.0\end{array}$ \\
\hline$H \rightarrow g g$ & 4.8 & $\begin{array}{l}+0.5 \\
-0.4 \\
\end{array}$ & $\begin{array}{l}+0.0 \\
+0.0\end{array}$ & $\begin{array}{l}+1.1 \\
+0.9\end{array}$ & $\begin{array}{l}+0.0 \\
+0.0\end{array}$ \\
\hline$H \rightarrow \tau^{+} \tau$ & 9.0 & $\begin{array}{l}+0.9 \\
-0.8 \\
\end{array}$ & $\begin{array}{l}+0.0 \\
+0.0 \\
\end{array}$ & $\begin{array}{l}+0.8 \\
-0.7 \\
\end{array}$ & $\begin{array}{l}+0.1 \\
\pm 0.1 \\
\end{array}$ \\
\hline
\end{tabular}

Table 4. Branching fractions (in \%) of an $80 \mathrm{GeV}$ Higgs boson evaluated from Eq. (12) with $M_{q}$ and $m_{q}\left(m_{H}\right)$, respectively, and evaluated including all channels and radiative corrections discussed in the text

\begin{tabular}{llll}
\hline Decay Mode & Tree-Level & $m_{q} \rightarrow m_{q}\left(m_{H}\right)$ & Full \\
\hline$H \rightarrow b \bar{b}$ & 87.3 & 85.5 & 83.3 \\
\hline$H \rightarrow \tau^{+} \tau^{-}$ & 4.2 & 11.8 & 9.0 \\
\hline$H \rightarrow c \bar{c}$ & 8.5 & 2.7 & 2.7 \\
\hline$H \rightarrow g g$ & - & - & 4.8 \\
\hline$H \rightarrow W^{*} W^{*}$ & - & - & 0.1 \\
\hline$H \rightarrow \gamma \gamma$ & - & - & 0.1 \\
\hline \hline
\end{tabular}

Zeitschrift für Physik C - Particles and Fields is delivered to some institutions in the former Soviet Union through a program initiated and administered by the European Physical Society and supported by the publisher. This program is funded by INTAS, the international association for the promotion of cooperation with scientists from the independent States of the former Soviet Union. Members of INTAS are the European Union, Austria, Belgium, Denmark, Finland, France, Germany, Greece, Ireland, Italy, Luxembourg, The Netherlands, Portugal, Spain, United Kingdom and Switzerland. (INTAS, rue du Luxembourg 14A, 1040 Bruxelles, Belgique). 